\title{
ON ABSOLUTE WEIGHTED MEAN SUMMABILITY METHODS
}

\author{
M. ALI SARIGÖL
}

(Communicated by Andrew M. Bruckner)

\begin{abstract}
In this paper we have proved the converse of the Bor-Thorpe theorem [2] on absolute weighted mean summability.
\end{abstract}

\section{INTRODUCTION}

Let $\sum a_{n}$ be a given infinite series with partial sums $s_{n}$, and let $A=\left(a_{n v}\right)$ be a normal matrix, that is, lower-semi matrix with nonzero entries. By $\left(A_{n}(s)\right)$ we denote the $A$-transform of the sequence $s=\left(s_{n}\right)$, i.e.,

$$
A_{n}(s)=\sum_{v=0}^{n} a_{n v} s_{v} .
$$

The series $\sum a_{n}$ is said to be summable $|A|_{k}, k \geq 1$, if

$$
\sum_{n=1}^{\infty}\left|a_{n n}\right|^{1-k}\left|A_{n}(s)-A_{n-1}(s)\right|^{k}<\infty,
$$

[4]. In the special case when $A$ is a Riesz matrix (resp. $k=1$ ), $|A|_{k}$ summability is the same as $\left|\bar{N}, p_{n}\right|_{k}$ (resp. $\left.\left|\bar{N}, p_{n}\right|\right)$ summability (see [1]). By a Riesz matrix we mean one such that

$$
\begin{aligned}
& a_{n v}=\frac{p_{v}}{P_{n}} \quad \text { for } 0 \leq v \leq n, \\
& a_{n v}=0 \quad \text { for } n>v,
\end{aligned}
$$

where $\left(p_{n}\right)$ is a sequence of positive real constants for which $P_{n}=p_{0}+p_{1}+$ $\cdots+p_{n} \neq 0, P_{n} \rightarrow \infty$ as $n \rightarrow \infty, P_{-1}=p_{-1}=0$.

If $P$ and $Q$ are methods of summability, $Q$ is said to include $P$ if every series summable by the method $P$ is also summable by the method $Q . P$ and $Q$ are said to be equivalent (written " $P \Leftrightarrow Q$ ") if each method includes the other.

Dealing with the absolute weighted mean summability of infinite series, Bor and Thorpe [2] established the following generalization of the Theorem due to Bor [1].

Received by the editors October 29, 1990.

1991 Mathematics Subject Classification. Primary 40F05, 40D25, 40G99. 
Theorem A. Suppose $\left(p_{n}\right)$ and $\left(q_{n}\right)$ are positive sequences such that

(i) $p_{n} Q_{n}=O\left(P_{n} q_{n}\right)$,

(ii) $P_{n} q_{n}=O\left(p_{n} Q_{n}\right)$.

Then $\left|\bar{N}, p_{n}\right|_{k} \Leftrightarrow\left|\bar{N}, q_{n}\right|_{k}, k \geq 1$.

\section{THE MAIN RESULT}

The object of this paper is to prove the converse of Theorem A as follows.

Theorem B. Suppose $\left(p_{n}\right)$ and $\left(q_{n}\right)$ are positive sequences. If $\left|\bar{N}, p_{n}\right|_{k} \Leftrightarrow$ $\left|\bar{N}, q_{n}\right|_{k}, k \geq 1$, then (1.1) holds.

For the proof of the theorem, we require the following

Lemma. Suppose that $k>0$ and $p_{n}>0, P_{n} \rightarrow \infty$ as $n \rightarrow \infty$. Then there exists two (strictly) positive constants $M$ and $N$, depending only on $k$, for which for all $v \geq 1$

$$
\frac{M}{P_{v-1}^{k}} \leq \sum_{n=v}^{\infty} \frac{p_{n}}{P_{n} P_{n-1}^{k}} \leq \frac{N}{P_{v-1}^{k}}
$$

where $M$ and $N$ are independent of $\left(p_{n}\right)$.

Proof. Consider the function $f:[0,1) \rightarrow \mathbb{R}$ given by $f(x)=(1-x)^{-1} \times$ $\left(1-x^{1 / k}\right)$. It is obvious that this is continuous and strictly positive. Also, $f(x) \rightarrow$ $1 / k$ as $x \rightarrow 1-0$. Thus there must be two positive constants $M$ and $N$, depending only on $k$, for which

$$
M \leq f(x) \leq N \quad \text { for all } 0 \leq x<1 .
$$

Now put $x=\left(P_{n-1} / P_{n}\right)^{k}=\left(1-p_{n} / P_{n}\right)^{k}$ in (2.2). Then we have, for $n \geq 1$,

$$
M\left(\frac{1}{P_{n-1}^{k}}-\frac{1}{P_{n}^{k}}\right) \leq \frac{p_{n}}{P_{n} P_{n-1}^{k}} \leq N\left(\frac{1}{P_{n-1}^{k}}-\frac{1}{P_{n}^{k}}\right) .
$$

Therefore (2.1) follows from (2.3), completing the proof of the lemma.

\section{Proof of Theorem B}

Let $\left(u_{n}(a)\right)$ and $\left(t_{n}(a)\right)$ be the sequences of $\left(\bar{N}, p_{n}\right)$ and $\left(\bar{N}, q_{n}\right)$ means of the series $a=\sum a_{n}$, respectively. Then by the definition, we have

$$
\begin{gathered}
u_{n}(a)=\frac{1}{P_{n}} \sum_{v=0}^{n} p_{v} s_{v}, \\
U_{n}(a)=u_{n}(a)-u_{n-1}(a)=\frac{p_{n}}{P_{n} P_{n-1}} \sum_{v=1}^{n} P_{v-1} a_{v}, \\
P_{-1}=0, \quad U_{0}(a)=a_{0},
\end{gathered}
$$


and

$$
\begin{gathered}
t_{n}(a)=\frac{1}{Q_{n}} \sum_{v=0}^{n} q_{v} s_{v}, \\
T_{n}(a)=t_{n}(a)-t_{n-1}(a)=\frac{q_{n}}{Q_{n} Q_{n-1}} \sum_{v=1}^{n} Q_{v-1} a_{v}, \\
Q_{-1}=0, \quad T_{0}(a)=a_{0} .
\end{gathered}
$$

For $k \geq 1$, define $\left|\bar{N}_{p}\right|_{k}=\left\{\left(a_{i}\right): \quad \sum a_{i}\right.$ is summable $\left.\left|\bar{N}, p_{n}\right|_{k}\right\}$ and $\left|\bar{N}_{q}\right|_{k}=$ $\left\{\left(a_{i}\right): \sum a_{i}\right.$ is summable $\left.\left|\bar{N}, q_{n}\right|_{k}\right\}$. Then it is routine to verify that these are $B K$-spaces if normed by

$$
\|a\|_{1}=\left\{\sum_{n=0}^{\infty}\left(\frac{P_{n}}{p_{n}}\right)^{k-1}\left|U_{n}(a)\right|^{k}\right\}^{1 / k}
$$

and

$$
\|a\|_{2}=\left\{\sum_{n=0}^{\infty}\left(\frac{Q_{n}}{q_{n}}\right)^{k-1}\left|T_{n}(a)\right|^{k}\right\}^{1 / k},
$$

respectively. Since $\left|\bar{N}, p_{n}\right|_{k} \Leftrightarrow\left|\bar{N}, q_{n}\right|_{k}$ by the hypothesis of the theorem, $\|a\|_{1}<\infty \Leftrightarrow\|a\|_{2}<\infty$, i.e., $\left|\bar{N}_{p}\right|_{k} \equiv\left|\bar{N}_{q}\right|_{k}$. Now consider the inclusion map $r:\left|\bar{N}_{p}\right|_{k} \rightarrow\left|\bar{N}_{q}\right|_{k}$ defined by $r(x)=x$ and its inverse. These are continuous, which is immediate as $\left|\bar{N}_{p}\right|_{k}$ and $\left|\bar{N}_{q}\right|_{k}, k \geq 1$, are $B K$-spaces. Thus there exists constants $H$ and $K$ such that

$$
H\|a\|_{1} \leq\|a\|_{2} \leq K\|a\|_{1}
$$

for all $a \in\left|\bar{N}_{p}\right|_{k} \equiv\left|\bar{N}_{q}\right|_{k}$. By applying (3.1) and (3.2) to $a=e_{v}-e_{v+1} \quad\left(e_{v}\right.$ is the $v$ th coordinate vector), respectively, we have

$$
\begin{aligned}
& U_{n}(a)= \begin{cases}0 & \text { if } n<v, \\
p_{v} / P_{v} & \text { if } n=v, \\
-p_{v} p_{n} / P_{n} P_{n-1} & \text { if } n>v\end{cases} \\
& T_{n}(a)= \begin{cases}0 & \text { if } n<v, \\
q_{v} / Q_{v} & \text { if } n=v, \\
-q_{v} q_{n} / Q_{n} Q_{n-1} & \text { if } n>v,\end{cases}
\end{aligned}
$$

so that (3.3) and (3.4) gives

$$
\begin{aligned}
& \|a\|_{1}=\left\{\frac{p_{v}}{P_{v}}+p_{v}^{k} \sum_{n=v+1}^{\infty} \frac{p_{n}}{P_{n} P_{n-1}^{k}}\right\}^{1 / k}, \\
& \|a\|_{2}=\left\{\frac{q_{v}}{Q_{v}}+q_{v}^{k} \sum_{n=v+1}^{\infty} \frac{q_{n}}{Q_{n} Q_{n-1}^{k}}\right\}^{1 / k} .
\end{aligned}
$$


Hence it follows from (3.5) that

$$
\begin{aligned}
H^{k}\left\{\frac{p_{v}}{P_{v}}+p_{v}^{k} \sum_{n=v+1}^{\infty} \frac{p_{n}}{P_{n} P_{n-1}^{k}}\right\} & \leq \frac{q_{v}}{Q_{v}}+q_{v}^{k} \sum_{n=v+1}^{\infty} \frac{q_{n}}{Q_{n} Q_{n-1}^{k}} \\
& \leq M^{k}\left\{\frac{p_{v}}{P_{v}}+p_{v}^{k} \sum_{n=v+1}^{\infty} \frac{p_{n}}{P_{n} P_{n-1}^{k}}\right\},
\end{aligned}
$$

which holds if and only if (1.1) is satisfied by the lemma. This completes the proof.

Our final result follows from Theorems A and B.

Theorem C. Suppose $\left(p_{n}\right)$ and $\left(q_{n}\right)$ are positive sequences with $P_{n} \rightarrow \infty$ and $Q_{n} \rightarrow \infty$ as $n \rightarrow \infty$. Then the necessary and sufficient condition for $\left|\bar{N}, p_{n}\right|_{k} \Leftrightarrow$ $\left|\bar{N}, q_{n}\right|_{k}, k \geq 1$, is that (1.1) hold.

We remark that if we take $q_{n}=1$ for all $n$, then $Q_{n}=n+1$. In this case $\left|\bar{N}, q_{n}\right|_{k}$ summability is the same as $|C, 1|_{k}$ summability [3]. Therefore the following corollary can be derived from Theorem $\mathrm{C}$.

Corollary. Necessary and sufficient conditions for $|C, 1|_{k} \Leftrightarrow\left|\bar{N}, p_{n}\right|_{k}$ are that

(i) $n p_{n}=O\left(P_{n}\right)$

(ii) $P_{n}=O\left(n p_{n}\right)$.

The sufficiency and necessity of this result are proven in [1] and [5], respectively.

\section{ACKNOWLEDGMENT}

The author is grateful to the referee for his kind remarks and suggestions that improved the presentation of the paper.

\section{REFERENCES}

1. H. Bor, A note on two summability methods, Proc. Amer. Math. Soc. 98 (1986), 81-84.

2. H. Bor and B. Thorpe, On some absolute summability methods, Analysis 7 (1987), 145-152.

3. T. M. Flett, On an extension absolute summability and some theorems of Littlewood and Paley, Proc. London Math. Soc. (3) 7 (1957), 113-141.

4. M. A. Sarigöl, On absolute summability factors, Comment. Math. Prac Mat. 31 (1991), (in press).

5. - Necessary and sufficient conditions for the equivalence of the summability methods $\left|\bar{N}, p_{n}\right|_{k}$ and $|C, 1|_{k}$, Indian J. Pure Appl. Math. 22 (6), (1991), 483-489.

Department of Mathematics, University of Erctyes, 38039 Kayseri, TURKey

E-mail address: sarigöl@trerun.bitnet 